Journal of

Bioinformatics and Comparative Genomics

\title{
Transcriptome, transcription factors and transcriptional regulation of leaf senescence
}

\author{
Wei Li and Yongfeng Guo*
}

Tobacco Research Institute, Chinese Academy of Agricultural Sciences, China

${ }^{*}$ Corresponding Author: Key Laboratory for Tobacco Gene Resources, Tobacco Research Institute, Chinese Academy of Agricultural Sciences, China; E-mail: guoyongfeng@caas.cn

Received Date: December 10, 2013 Accepted Date: January 21, 2014 Published Date: February 06, 2014

Citation: Yongfeng Guo (2014) Transcriptome, transcription factors and transcriptional regulation of leaf senescence. J Bioinfo Comp Genom 1: 1-3

\begin{abstract}
Senescence is the last stage of development of a leaf, and is critical for plants' evolutionary fitness as nutrient remobilization to other organs of a plant is achieved through this process. The leaf senescence process is believed to be under the control of a highly regulated genetic program which is associated with drastic changes of gene expression. Major breakthroughs in the molecular understanding of leaf senescence have been achieved through analyses of the transcriptome and transcriptional regulations during this process. Changes in gene expression reflect the profound physiological rearrangements and studies of transcription factors have provided intriguing clues for the regulatory network involved. This review summarizes recent progress in transcriptomic analyses and studies on transcriptional regulation of leaf senescence.

Keywords: Leaf senescence; Transcriptome; Gene expression; Transcription factors.
\end{abstract}

Senescence is the age-dependent ending of a life cycle. In plants, senescence is characterized by the visible yellowing of leaves. The yellowing of senescing leaves is correlated with a series of biochemical changes such as loss of chlorophyll contents, degradation of proteins and RNA, and a decline in photosynthetic activity. All of these substantial changes are associated with alterations in gene expression. Many genes expressed in green leaves, such as the genes encoding photosynthetic enzymes, are downregulated upon the onset of senescence. Conversely, many senescence-associated genes (SAGs) are transcriptionally upregulated during leaf senescence $[1,2]$ (Figure 1). Identification and characterization of SAGs is critical in understanding the biochemical and physiological processes of leaf senescence.

Clarification of the molecular mechanisms underlying senescence has been facilitated by cloning and characterization of $S A G s$ isolated using forward and reverse genetic approaches $[3,4]$. Traditional molecular biology approaches in which one gene or mutant at a time is identified and analyzed focus on individual molecules such as nucleotide acids, proteins, and metabolites. These researches thus have been limited to a

(C)2013 The Authors. Published by the JScholar under the terms of the Creative Commons Attribution License http://creativecommons.org/licenses/ by $/ 3.0 /$, which permits unrestricted use, provided the original author and source are credited. handful of genes and have generally failed to reveal a global picture of senescence regulatory networks.

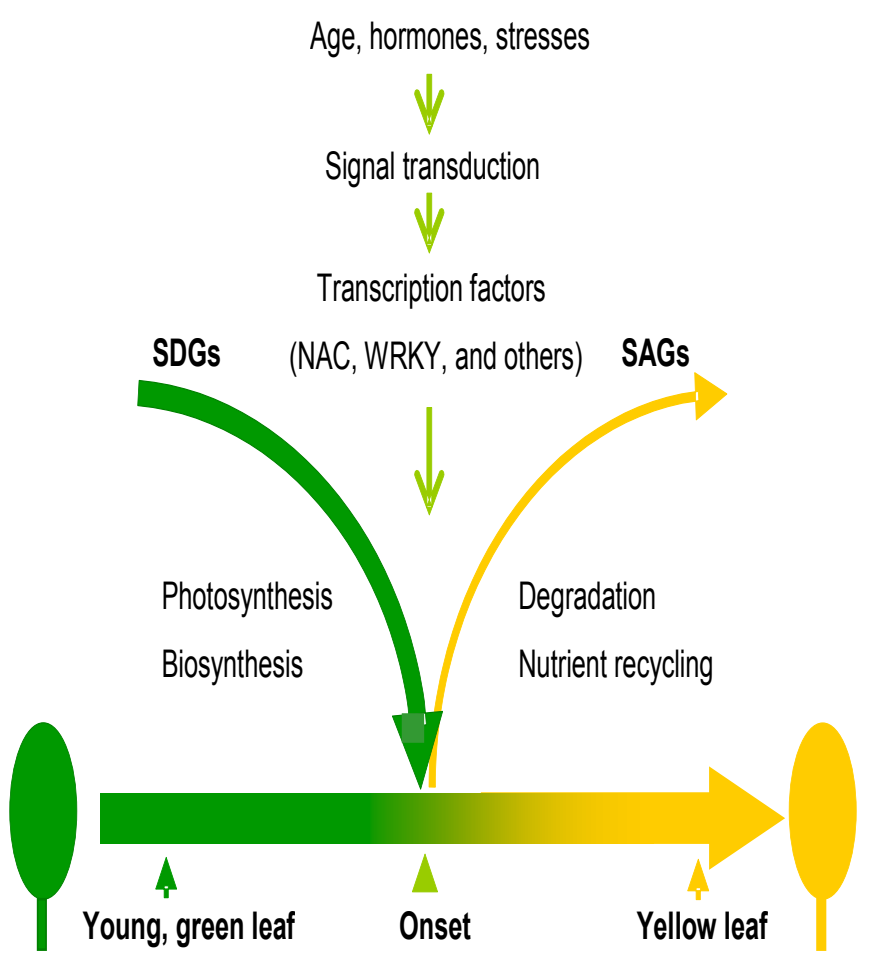

Figure 1 Transcriptional regulation of leaf senescence 
With the advancements in genome sequencing and whole transcriptome profiling tools, a number of studies have investigated the global transcriptional reprogramming during natural and induced senescence $[5,6]$. Guo et al. have revealed 2491 genes expressed at the latest stage of leaf development through single-pass sequencing of a senescent leaf cDNA library. Among these genes, there are 134 genes encoding transcription factors and 182 genes whose products participate in signal transduction pathways, including receptor-like kinases and components of the mitogen-activated protein kinase (MAPK) cascades. A total of 116 genes encode enzymes involved in breaking down proteins, such as components in the ubiquitin-proteasome pathway and proteinases. Furthermore, probably due to the fact that gene expression profiles in senescent tissues are rarely studied before, this study identified ESTs from 98 annotated genes for the first time and 46 novel transcripts that have not previously been annotated in the Arabidopsis genome [7].

Microarray-based expression profiling is the most widely used approach in measuring the expression levels of large numbers of genes simultaneously. Microarray analysis of Arabidopsis cDNAs by Zentgraf et al. has shown changes in the expression of about $20 \%$ of all the genes during leaf senescence [8]. Subsequently, through microarray analysis using the Affymetrix Arabidopsis whole genome GeneChip (ATH1), BuchananWollaston et al. identified 827 SAGs that showed at least a threefold upregulation upon leaf senescence [9]. In a more recent study, Breeze et al. analyzed a high-resolution timecourse profile of gene expression of Arabidopsis leaves before and after the onset of senescence [10]. This study categorized 6,323 differentially expressed genes into 48 clusters based on patterns of gene expression changes at 22 time points. With the major switch in gene expression coinciding with the appearance of visible yellowing, successive changes in gene expression reflected the major molecular events that take place during the process of leaf senescence. Signaling of senescence-promoting hormones, downregulation of chlorophyll biosynthesis, and upregulation of senescence-associated transcription factors are among the earliest events that happened during leaf senescence, followed by the degradative processes and declines of photosynthetic activities. With the progression of leaf yellowing, more catalytic activities for catabolism were detected, which were accompanied by the enrichment of transport activities, probably indicating nutrient remobilization [10]. Leaf senescence can be induced by many hormonal and environmental factors. In order to compare gene expression changes induced by developmental senescence and other senescence-inducing factors, Guo and Gan analyzed microarray data from 27 different senescence-inducing treatments and developmental leaf senescence. Results from this study indicated that although gene expression profiles from different treatments differed significantly at early stages, after a prolonged period of treating time, generally after visible senescence is induced, significant overlap in gene expression changes were observed between developmental leaf senescence and different senescence-inducing treatments. This suggests that different senescence-inducing signals may promote the initiation of senescence through different signaling pathways but the common senescence execution processes might be shared by senescence induced by different signals [6].

RNA-seq has been increasingly used for global gene expression profiling as it allows unbiased quantification of expression levels of transcripts with a higher sensitivity and broader genome coverage than microarrays [11]. An analysis by Sîrbu et al. compared gene expression data of single/dual-channel microarrays and RNA-seq for Drosophila melanogaster embryo development time series. This study revealed that the RNA-seq technique is better in detecting transcripts with low abundance and as a result, 2000 more differentially expressed genes were identified with RNA-seq [12]. Using the Solexa sequencing system, Kong et al. compared the difference between the transcriptomes of age-matched leaves from an early senescence (K1) and a late senescence (K2) cotton line at late stages of leaf development [13]. The profiling analysis indicated that 1132 genes were upregulated and 455 genes downregulated in $\mathrm{K} 1$ compared with $\mathrm{K} 2$. Among the upregulated genes were genes related to catabolism of proteins, nucleic acids, lipids and genes involved in nutrient recycling. Genes predicted to function in photosynthesis, anabolism of carbohydrates and other biomolecules on the other hand, were downregulated in the early senescence line. This study also identified 11 NAC and WRKY transcription factor genes that were upregulated during leaf senescence [13].

The drastic change in gene expression is usually driven by transcription factors, which are master-control proteins regulating activation/ suppression of gene expression through binding to specific regulatory sequences of target genes. Various transcriptomic analyses have identified NAC, WRKY, AP2/EREBP, MYB, C2H2 zinc-finger, bZIP, and GRAS being the largest families of transcription factors that are upregulated during leaf senescence $[7,9,14-16]$, among which NAC and WRKY genes have been widely investigated for their regulatory roles in leaf senescence.

The functions of a number of NAC and WRKY genes have been investigated in detail. The AtNAP gene, encoding one of the NAC family transcription factors, has been shown to be transcriptionally upregulated during senescence of Arabidopsis leaves [17]. Overexpression of AtNAP led to premature senescence, whereas the atnap knockout mutant plants exhibited a delayed leaf senescence phenotype. Furthermore, NAP genes from rice and kidney bean, OsNAP and PvNAP, restored the delayed leaf senescence in the atnap null plants, showing that the NAP genes are conserved in different plant species [17]. Downstream targets of transcription factor AtNAP include SAG113, a protein phosphatase 2C-encoding gene which functions to regulate ABA-mediated stomatal movement and water loss during leaf senescence [18, 19]. The NAC family member AtORE1/ANAC092 has also been identified as a positive regulator of senescence [20]. 170 genes that were significantly induced and 48 genes downregulated by ANAC092 had been identified through microarray-based expression profiling as potential target genes [21]. ABA responsive NAC transcription factor VNI2 can integrate ABAmediated abiotic stress signals into leaf aging by regulating 
a subset of COR (COLD-REGULATED) and RD (RESPONSIVE TO DEHYDRATION) genes [22]. In addition, NAC family transcription factors ORS1 [23] and NTL4 [24] play a role in promoting senescence in Arabidopsis while JUB1 is a negative regulator of senescence [25].

Like NACs, WRKY transcription factors also play a central role in controlling leaf senescence in plants $[26,27]$. WRKY6 [28], WRKY22 [29], WRKY53 [30], WRKY54 and WRKY70 [31] are all involved in leaf senescence regulation. The most well studied WRKY53 is a positive senescence regulator. WRKY53 knock-out plants show a delayed senescence phenotype [30]. Expression of WRKY53 is upregulated at an early stage then down-regulated again at later stages of leaf senescence. WRKY53 is thus believed to play a regulatory role in the early events of senescence [32]. Sixty three genes, including at least six other members of the WRKY gene family, have been isolated as putative target genes of WRKY53 via genomic pull down assays, suggesting that this WRKY transcription factor acts as an upstream control element in a transcription factor signaling cascade leading to leaf senescence [30].

As a complex process regulated by multiple endogenous and environmental factors, leaf senescence involves expression changes of more than one quarter of all Arabidopsis genes. Transcriptomic analyses in combination with analyses of key transcription factors have started unveiling the global transcription regulatory network underlying leaf senescence. This regulatory network includes not only a global picture of all the signaling and biochemical events that occur during leaf senescence, but also the integrated connections and detailed crosstalk between these events. More in depth studies based on known senescence-regulating transcription factors will facilitate further understanding of the systematic regulation of gene expression during leaf senescence.

\section{Acknowledgements}

Research in the Guo Lab is supported by China's Ministry of Agriculture (2013-Z4), Chinese Academy of Agricultural Sciences (CAAS) (2013ZL024), and the Tobacco Research Institute, CAAS.

\section{References}

1) YF Guo, SS Gan (2005) Leaf senescence: Signals, execution, and regulation. Current topics in developmental biology 71: 83-112.

2) PO Lim, HJ Kim, HG Nam (2007) Leaf Senescence. Annu Rev Plant Biol 58: $115-136$.

3) H Zhang, C Zhou (2013) Signal transduction in leaf senescence. Plant Mol Biol 82: 539-545.

4) R Jibran, A Hunter D, P Dijkwel P (2013) Hormonal regulation of leaf senescence through integration of developmental and stress signals. Plant Mol Biol 82: 547-561.

5) Y Guo (2013) Towards systems biological understanding of leaf senescence. Plant Mol Biol 82: 519-528.

6) Y Guo, Gan SS (2012) Convergence and divergence in gene expression profiles induced by leaf senescence and 27 senescence-promoting hormonal, pathological and environmental stress treatments. Plant Cell Environ 35: 644655 .

7) Y Guo, Z Cai, S Gan (2004) Transcriptome of Arabidopsis leaf senescence. Plant Cell Environ 27: 521-549.
8) U Zentgraf, J Jobst, D Kolb, D Rentsch (2004) Senescence-Related Gene Expression Profiles of Rosette Leaves of Arabidopsis thaliana: Leaf Age Versus Plant Age. Plant Biology 6: 178-183.

9) V Buchanan-Wollaston, T Page, E Harrison, E Breeze, PO, Lim et al. (2005) Comparative transcriptome analysis reveals significant differences in gene expression and signalling pathways between developmental and dark/starvation-induced senescence in Arabidopsis. Plant J 42: 567-585.

10) E Breeze, E Harrison, S McHattie, L Hughes, R Hickman, et al. (2011) High-Resolution Temporal Profiling of Transcripts during Arabidopsis Leaf Senescence Reveals a Distinct Chronology of Processes and Regulation. Plant Cell 23: 873-894.

11) A Mortazavi, BA Williams, K McCue, L Schaeffer, B Wold (2008) Mapping and quantifying mammalian transcriptomes by RNA-Seq. Nature methods 5: 621-628.

12) A Sîrbu, G Kerr, M Crane, HJ Ruskin (2012) RNA-Seq vs Dual- and Single-Channel Microarray Data: Sensitivity Analysis for Differential Expression and Clustering. PloS one 7: e50986.

13) $X$ Kong, Z Luo, H Dong, AE Eneji, Li W et al (2013) Gene expression profiles deciphering leaf senescence variation between early-and late-senescence cotton lines. PloS one 8: e69847.

14) PL Gregersen, PB Holm (2007) Transcriptome analysis of senescence in the flag leaf of wheat (Triticum aestivum L.). Plant Biotechnol J 5: 192-206.

15) S Balazadeh, DM Riano-Pachon, B Mueller-Roeber (2008) Transcription factors regulating leaf senescence in Arabidopsis thaliana. Plant Biol (Stuttg) 1: 63-75.

16) ST Shah, C Pang, S Fan, M Song, S Arain, et al. (2013) Isolation and expression profiling of GhNAC transcription factor genes in cotton (Gossypium hirsutum L.) during leaf senescence and in response to stresses. Gene 531: 220-234.

17) Y Guo, S Gan (2006) AtNAP, a NAC family transcription factor, has an important role in leaf senescence. Plant J 46: 601-612.

18) K Zhang, X Xia, Y Zhang, SS Gan (2012) An ABA-regulated and Golgilocalized protein phosphatase controls water loss during leaf senescence in Arabidopsis. Plant J 69: 667-678.

19) K Zhang, SS Gan (2012) An abscisic acid-AtNAP transcription factorSAG113 protein phosphatase $2 \mathrm{C}$ regulatory chain for controlling dehydration in senescing Arabidopsis leaves. Plant Physiol, 158: 961-969.

20) JH Kim, HR Woo, J Kim, PO Lim, IC Lee, et al. (2009) Trifurcate feedforward regulation of age-dependent cell death involving miR164 in Arabidopsis. Science 323: 1053-1057.

21) S Balazadeh, H Siddiqui, AD Allu, LP Matallana-Ramirez, C Caldana, et al. (2010) A gene regulatory network controlled by the NAC transcription factor ANAC092/AtNAC2/ORE1 during salt-promoted senescence. Plant J 62: 250-264.

22) SD Yang, PJ Seo, HK Yoon, CM Park (2011) The Arabidopsis NAC transcription factor VNI2 integrates abscisic acid signals into leaf senescence via the COR/RD genes. The Plant Cell Online 23: 2155-2168.

23) S Balazadeh, M Kwasniewski, C Caldana, M Mehrnia , MI Zanor, et al. (2011) ORS1, an $\mathrm{H}(2) \mathrm{O}(2)$-responsive NAC transcription factor, controls senescence in Arabidopsis thaliana. Molecular plant 4: 346-360.

24) S Lee, PJ Seo, HJ Lee, CM Park (2012) A NAC transcription factor NTL4 promotes reactive oxygen species production during drought-induced leaf senescence in Arabidopsis. Plant J 70: 831-844.

25) A Wu, AD Allu, P Garapati, H Siddiqui, H Dortay, et al. (2012) JUNGBRUNNEN1, a reactive oxygen species-responsive nac transcription factor, regulates longevity in Arabidopsis. The Plant Cell Online 24: 482-506.

26) PJ Rushton, IE Somssich, P Ringler, QJ Shen (2010) WRKY transcription factors. Trends Plant Sci 15: 247-258.

27) U Zentgraf, T Laun, Y Miao (2010) The complex regulation of WRKY53 during leaf senescence of Arabidopsis European journal of cell biology 89: 133-137.

28) S Robatzek, IE Somssich (2001) A new member of the Arabidopsis WRKY transcription factor family, AtWRKY6, is associated with both senescenceand defence-related processes. Plant J 28: 123-133. 
29) X Zhou, Y Jiang, D Yu (2011) WRKY22 transcription factor mediates dark-induced leaf senescence in Arabidopsis. Mol Cells 31: 303-313.

30) Y Miao, T Laun, P Zimmermann, U Zentgraf (2004) Targets of the WRKY53 transcription factor and its role during leaf senescence in Arabidopsis. Plant Mol Biol 55: 853-867.
31) S Besseau, J Li, ET Palva (2012) WRKY54 and WRKY70 co-operate as negative regulators of leaf senescence in Arabidopsis thaliana. J Exp Bot 63: 2667-2679.

32) K Hinderhofer, U Zentgraf (2001) Identification of a transcription factor specifically expressed at the onset of leaf senescence. Planta 213: 469-473.

Submit your manuscript to JScholar journals and benefit from:

- Convenient online submission

ฯ Rigorous peer review

q Immediate publication on acceptance

- Open access: articles freely available online

9 High visibility within the field

9 Better discount for your subsequent articles

Submit your manuscript at http://www.jscholaronline.org/submit-manuscript.php 